Gut, 1965, 6, 80

\title{
Reflux after cardiomyotomy
}

\author{
FRANK ELLIS AND F. L. COLE \\ From the Departments of Surgery and Radiology, Guy's Hospital, London
}

EDITORIAL SYNOPSIS A series of 56 patients with achalasia of the cardia included 16 with reflux after operation of whom seven were symptomless. A radiological technique which facilitated the detection of reflux was employed.

The factors contributing to the development of reflux included duodenal ulceration, previous oesophageal operations, double and strip myotomies, and disruption of the hiatus. Of nine patients with reflux oesophagitis, five required further operative treatment. Careful pre-operative evaluation and the preservation of the hiatal mechanism are considered to be the most important factors in reducing the incidence of reflux. The long myotomy is considered to be necessary to ensure adequate oesophageal drainage. If it is placed on the lesser curve side of the oesophagus and stomach the risk of reflux is likely to be diminished.

The return of achalasia-like symptoms after cardiomyotomy may be due either to an inadequate division of the circular muscle fibres of the lower segment or to gastro-oesophageal reflux through a cardia rendered incompetent by the operation and thereby allowing oesophagitis, ulceration, and fibrosis to occur. Although the incidence of postoperative gastro-oesophageal reflux varies widely, there is general agreement that it is important to distinguish between a true recurrence of the achalasia and reflux oesophagitis because the two conditions need different management. Douglas and Nicholson (1959) found two reflux strictures among 41 cases of cardiomyotomy whereas Nemir and Frobese (1962) recommended the addition of pylorotomy to cardiomyotomy following an incidence of $40 \%$ reflux among their early cases. Atkinson (1959) investigated the pressure in the lower oesophageal segments of 18 patients who had been subjected to cardiomyotomy and in whom subsequent reflux was suspected on clinical grounds. In the majority the resting pressure was found to be lower than normal but only five had demonstrable reflux. Among 61 patients subjected to cardiomyotomy Barlow (1961) found 15 with some degree of reflux and six with strictures.

A long pre-operative history of oesophageal symptoms is often present in patients subjected to cardiomyotomy. If, after the operation, reflux produces further symptoms they do not tend to complain as readily as those suffering from such symptoms for the first time. The evasive features of the clinical picture are further complicated by difficulty in detecting reflux radiologically or endoscopically. This difficulty is primarily due to the fact that, despite treatment, the oesophagus in achalasia not uncommonly contains some residue. Unless special measures are taken to empty the dilated segment, the contrast material remaining above the cardia following a barium meal will obscure the region to such an extent that reflux from the stomach may not be seen. In the same way the detection of reflux on oesophagoscopy will be hampered by the presence of residue and by the mechanical difficulties of visualizing the lower end of the oesophagus.

This paper reports the results of an investigation into the incidence, causes, and effects of reflux following cardiomyotomy. From 1943 to 1963 cardiomyotomy was carried out in 56 patients. Table I shows the status of these patients in 1963. Forty-five were reviewed clinically and radiologically in 1963, five were last reviewed similarly in 1956, three were dead, and three untraced. Of the total of 56, 17 were found to have radiological reflux. One of the patients who died developed a gross stricture following first a Mikulicz operation and then a cardiomyotomy. Finally her symptoms

\section{TABLE I}

STATUS OF 56 PATIENTS SUBJECTED TO CARDIOMYOTOMY DURING 1943-1963

\begin{tabular}{lcc} 
Follow-up & No. of Cases & No. With Reflux \\
\hline To 1963 & 45 & 15 \\
To 1956 only & 5 & 1 \\
Dead & 3 & 1 \\
No follow-up & 3 & -17 \\
Total & 56 & 17
\end{tabular}


were relieved for three years before her death by ileocaecal transposition.

The mean interval from the time of cardiomyotomy to follow-up was eight years, the range being two to 20 years. Most of the operations were carried out through the chest and in most cases a 'long' single anterior myotomy was extended from the oesophagus on to the stomach.

\section{DETECTION OF REFLUX}

RADIOLOGICAL TECHNIQUE The symptoms of reflux in these patients proved to be so diverse that attempts to forecast its presence before screening were, except in gross cases, highly inaccurate. Using image intensification and television screening a radiological technique was designed to overcome the difficulties of visualizing gastroesophageal incompetence in the presence of incomplete emptying of the oesophagus.

After preliminary fasting each patient was given a third of a litre of barium and 10 minutes later the oesophagus was screened. While many of the patients had little or no oesophageal delay, in nearly half there remained in the oesophagus sufficient barium to interfere with the detection of reflux by the usual methods. If sufficient time was allowed to elapse in order to allow the oesophagus to empty, most of the barium was found to have left the stomach and insufficient remained to detect reflux. This difficulty was overcome by giving each patient water to drink until the residual barium passed into the stomach leaving the lower oesophagus radiotranslucent. In most cases about one-third of a litre of water was necessary and the oesophagus was free of barium within a few moments. The patient was then placed in the Trendelenburg position and reflux of barium was sought by applying external pressure over the stomach with the patient first supine and then prone. Figure 1a shows a residual column of barium in a patient entirely free of symptoms eight years after cardiomyotomy. After drinking water (Fig. 1b) the oesophagus was virtually empty. When the patient was tilted head-down the reflux which occurred (Fig. 1c) was readily detectable whereas it could easily have escaped notice if the barium residue had still been present.

The second point in the technique is one for which we are indebted to Mr. Hermon-Taylor. It was found that several of the patients in whom no reflux occurred when the usual postural methods were used, had a slow 'trickle-back' of barium which was only revealed when they were allowed to relax for 10 minutes in the Trendelenburg position. By using these two additions to the usual methods of radiological detection of reflux, gastroesophageal incompetence which would have otherwise escaped notice was detected. Of the $\mathbf{4 5}$ patients screened in 1963,15 were found to have reflux.

CLINICAL FEATURes Without prior knowledge of the radiological appearances all the patients were interviewed by one observer. They were questioned closely about the symptoms of heartburn and regurgitation both before and after cardiomyotomy. The patients were then screened by the other observer without detailed knowledge of the clinical features. Having established the radio logical competence of the gastro-oesophageal sphincter the clinical features of the whole group were reviewed with special reference to symptoms of reflux. Two anomalies were found. 1 Among 30 patients without reflux, eight had occasional heartburn and regurgitation. 2 Among 16 patients having reflux (Table II), seven had no symptoms and were classed as having good or excellent results from the operation, five had pain and regurgitation while the remaining four had gross strictures accompanied by all the features of oesophageal obstruction.

\section{RESULTS}

These findings suggest that heartburn and regurgitation, the usual hallmarks of gastro-oesophageal incompetence, are not reliable indicators of reflux in patients with achalasia. The lack of correlation between clinical and radiological findings is a wellknown feature of this disease in all its stages, and the data in this group of patients emphasize that, for the proper evaluation of the gastro-oesophageal junction after cardiomyotomy, there is no substitute for careful radiology.

FACTORS PREDISPOSING TO REFLUX Table II shows that in eight of 16 patients with radiological reflux,

TABLE II

CLINICAL FEATURES OF PATIENTS WITH RADIOLOGICAL REFLUX AFTER CARDIOMYOTOMY

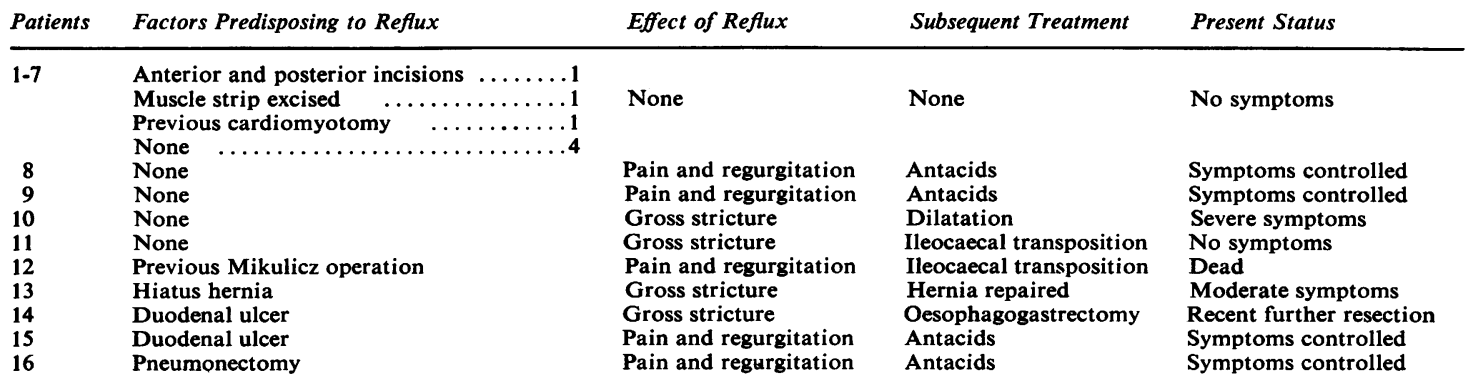




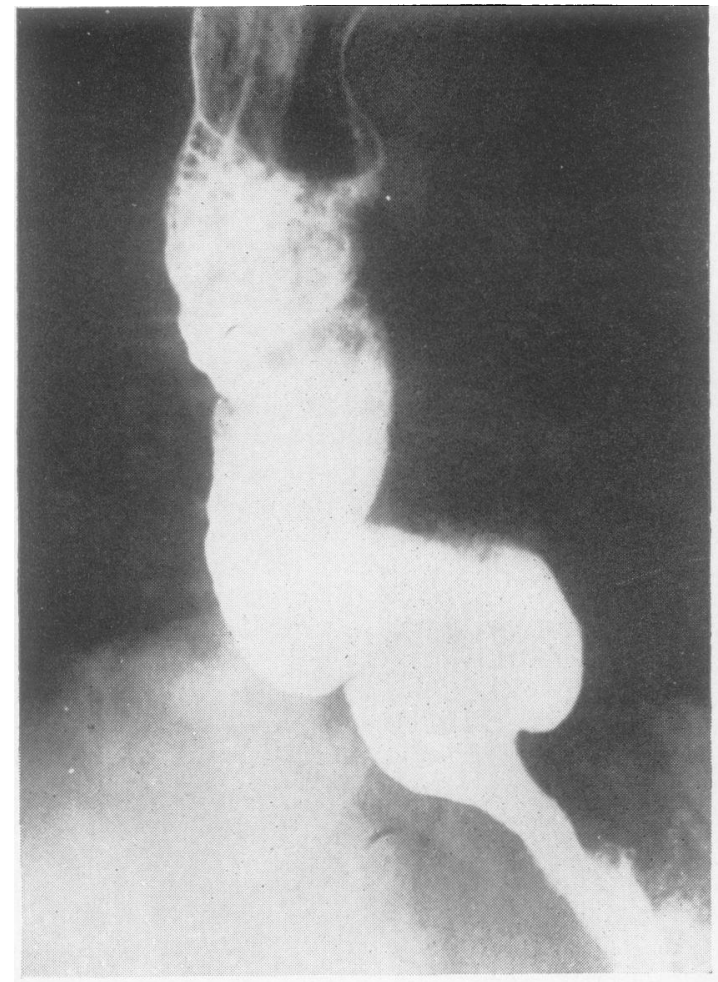

FIG. 1a.
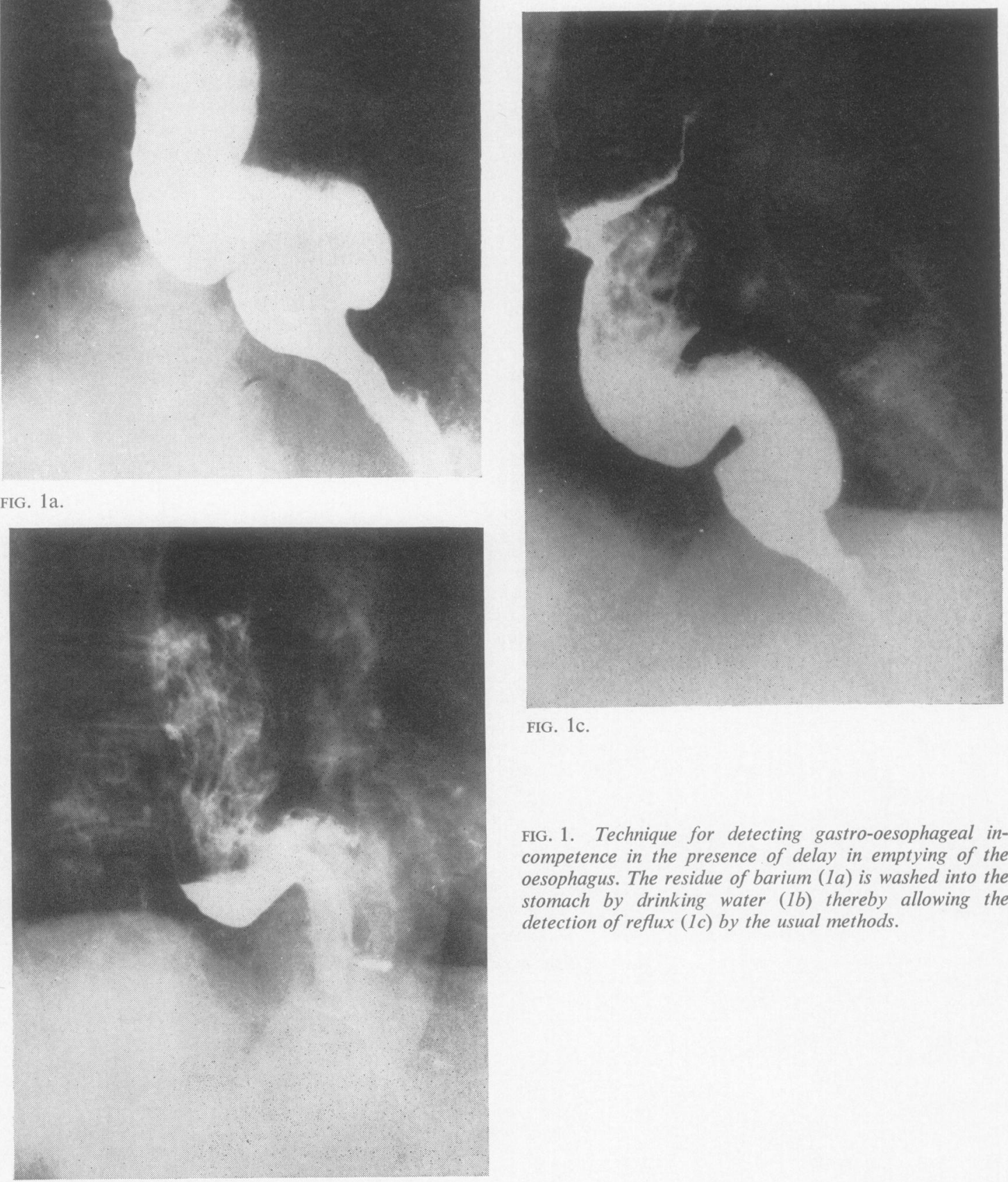

FIG. 1c.

FIG. 1. Technique for detecting gastro-oesophageal incompetence in the presence of delay in emptying of the oesophagus. The residue of barium (1a) is washed into the stomach by drinking water $(1 b)$ thereby allowing the detection of reflux (1c) by the usual methods.

FIG. 1b. 


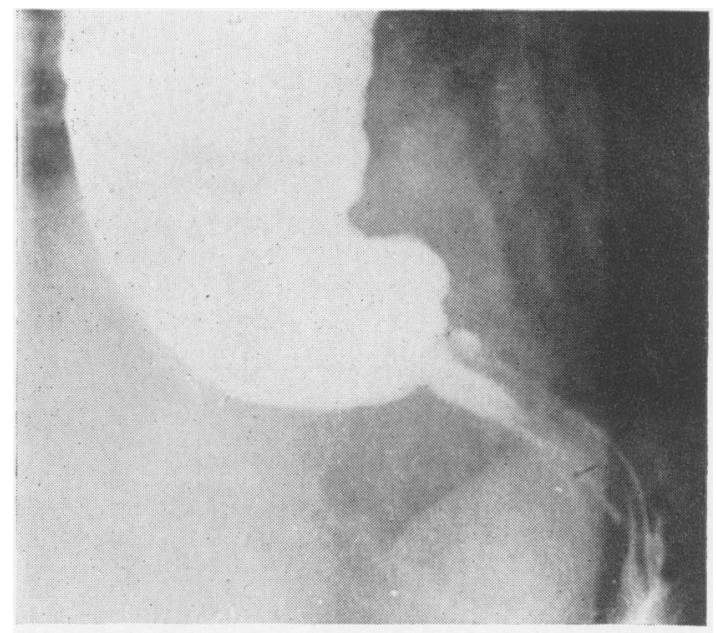

FIG. $2 \mathrm{a}$.

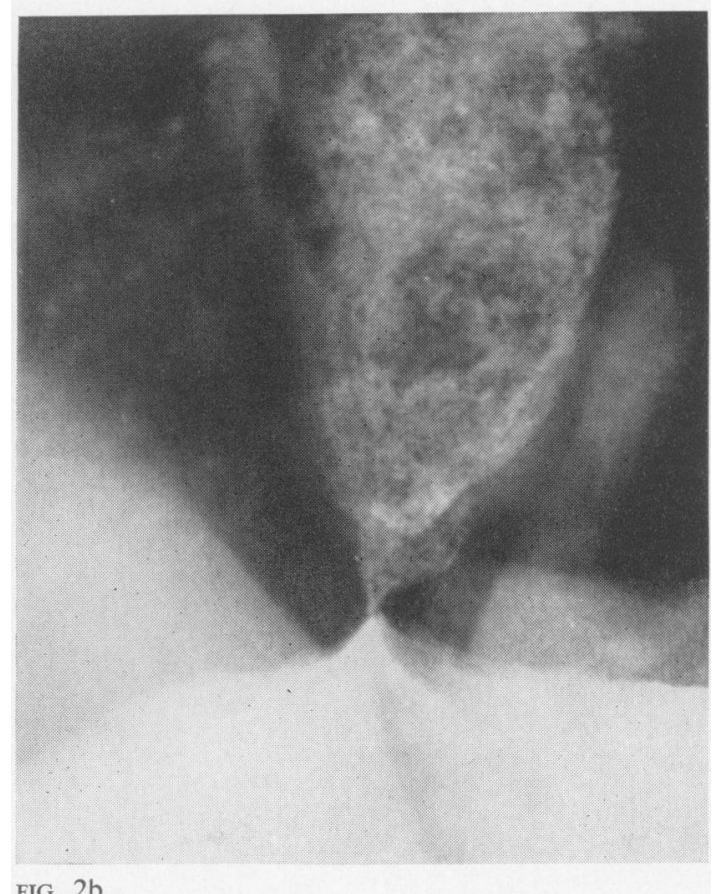

FIG. 2. The effects of reflux after cardiomyotomy in a patient with achalasia who also had a duodenal ulcer. An ulcer crater (2a) developed and was followed by complete oesophageal stenosis $(2 b)$.

there were possible explanations for the presence of reflux after cardiomyotomy. Four patients had more than the modified Heller's operation which is performed at present. Of these one had anterior and posterior incisions, one a strip of muscle excised, and two had been subjected to previous sphincterdestroying operations. Of the four remaining patients two had duodenal ulcers, one a pneumonectomy, and one a hiatus hernia.

SUBSEQUENT TREATMENT AND PROGRESS Seven patients had no symptoms and were classed as having good or excellent results. Four had moderate symptoms which were controlled by antacids. Five required further surgical treatment having developed severe oesophageal obstruction. They were subjected to a variety of procedures (Table II). In all cases, except case 11 in whom no symptoms are present but reflux still occurs freely, the results were unsatisfactory and having each had two operations these patients were more severely incapacitated than by the original achalasia. Figure $2 \mathrm{a}$ shows an oesophageal ulcer which developed with reflux three years after cardiomyotomy. The patient was subsequently shown to have a duodenal ulcer. Later (Fig. 2b) gross oesophageal stenosis occurred.

\section{DISCUSSION}

Among the 45 patients followed to 1963 there were 12 in whom the results of cardiomyotomy were unsatisfactory. In no less than seven of these reflux was responsible for the recurrence. This means that a substantial proportion of the failures following cardiomyotomy could be eliminated by the prevention of post-operative reflux. However, since seven patients with reflux had no symptoms and have remained well for many years following operation, it is evident that reflux and reflux oesophagitis are not synonymous.

The problem of reflux after cardiomyotomy appears in some respects to be a surgical impasse because an operation which produces adequate oesophageal drainage may so lower the competence of the gastro-oesophageal sphincter that reflux is inevitable. This is more likely to occur in those patients in whom the lower oesophageal sphincter (as opposed to the other mechanisms) is the most important factor contributing to competence. An operation which fails to achieve complete division of the circular muscle will almost certainly not ensure adequate oesophageal drainage though reflux is less likely to occur. This impasse may be partly resolved by careful selection of patients and by careful attention to detail during operation.

SELECTION OF PATIENTS The experience from this group of patients shows that the only two patients with duodenal ulcers developed reflux oesophagitis. It is important to avoid cardiomyotomy in such patients, particularly if they have gastric retention. 
In addition to pre-operative radiological examination for duodenal ulcer, it may also be advisable to estimate the acid production of the stomach. If this is high, even in the absence of duodenal ulceration, it is possible that if reflux occurs after cardiomyotomy oesophagitis will tend to develop more readily than in a patient with normal acid production. The presence of inflammatory changes in the oesophageal mucosa should arouse suspicion that reflux may be present even before operation and the patient investigated accordingly. The previous exploration of the lower oesophageal segment which had been carried out in two of the patients with reflux suggests that incompetence may occur more readily after such procedures.

OPERATIVE TECHNIQUE McVey, Schlegel, and Ellis (1963) have shown that in animals the characteristic high pressure zone in the lower segment is diminished or abolished following cardiomyotomy. Atkinson (1959) has shown a similar effect in man. The effect in animals is greater with the classical double Heller myotomy and with the 'long' myotomy than with the 'short' in which the incision is stopped at the oesophago-gastric junction. However, there is likely to be less adequate drainage with a short incision. It is possible that the free reflux which may occur with the long incision extended on to the stomach is due to division of the oblique sling of muscle fibres (sling of Helvetius) which help to maintain the mucosal rosette. The fibres can be avoided by making the incision on the right side of the oesophagus and extending it down the lesser curve rather than on the anterior surface of the stomach.

A most important operative principle in maintaining the delicate balance between procedures which ensure free oesophageal drainage without allowing reflux to occur and those which result in gastro-oesophageal incompetence is the preservation of the hiatal mechanism. Excessive mobilization of the oesophagus, division of the phreno-oesophageal ligaments, displacement of the stomach into the chest, and failure to restore the lower segment of the oesophagus to its position below the diaphragm all contribute to weaken the hiatal mechanism thereby producing sufficient imbalance to allow reflux to occur. If such defects are unavoidable a repair should be carried out after the myotomy has been established. Avoidance of damage to the vagus is imperative because post-operative gastric retention may result in reflux. In view of the accumulating evidence that reflux occurs alongside nasogastric suction tubes these are also probably best avoided.

Finally it has been suggested that cardiomyotomy should be accompanied by additional procedures to prevent reflux. Suturing the fundus of the stomach to the diaphragm (Barlow, 1961), pyloroplasty, and gastroenterostomy (Hawthorne, Frobese, and Nemir 1956) have been described. It would appear that these additions to the operation impose an unnecessarily heavy burden on the patient, but they may merit attention where duodenal ulceration and achalasia coexist. For the remainder, careful attention to operative detail will do much to reduce the small proportion of unsatisfactory results which follow cardiomyotomy. Radiological assessment in the immediate post-operative period and after an interval of six months will probably reveal a small number in whom reflux occurs despite adequate precautions.

These patients need long-term supervision before it is evident how much difficulty the reflux will cause.

\section{REFERENCES}

Atkinson, M. (1959). The oesophago-gastric sphincter after cardiomyotomy. Thorax, 14, 125-131.

Barlow, D. (1961). Problems of achalasia. Brit. J. Surg., 48, 642-645.

Douglas, K., and Nicholson, F. (1959). The late results of Heller's operation for cardiospasm. Ibid., 47, 250-253.

Hawthorne, H. R., Frobese, A. S., and Nemir, P., Jr. (1956). The surgical management of achalasia of the esophagus. Ann. Surg., 144, 653-669.

McVey, J. L., Schlegel, J. F., and Ellis, F. H., Jr. (1963). Gastroesophageal sphincteric function after the Heller myotomy and its modifications. Bull. Soc. int. Chir., 22, 419-423.

Nemir, P., Jr., and Frobese, A. S. (1962). The modified Heller operation for achalasia of the esophagus. Surg. Clin. N. Amer., 42, 1407-1418. 